\title{
Delft University of Technology
}

\section{Asymmetric vortex shedding in the wake of an abruptly expanding annular jet}

Vanierschot, Maarten; Percin, Mustafa; van Oudheusden, Bas W.

DOI

10.1007/s00348-021-03177-9

Publication date

2021

Document Version

Final published version

Published in

Experiments in Fluids

\section{Citation (APA)}

Vanierschot, M., Percin, M., \& van Oudheusden, B. W. (2021). Asymmetric vortex shedding in the wake of an abruptly expanding annular jet. Experiments in Fluids, 62(4), [77]. https://doi.org/10.1007/s00348-02103177-9

\section{Important note}

To cite this publication, please use the final published version (if applicable).

Please check the document version above.

\section{Copyright}

Other than for strictly personal use, it is not permitted to download, forward or distribute the text or part of it, without the consent of the author(s) and/or copyright holder(s), unless the work is under an open content license such as Creative Commons.

\section{Takedown policy}

Please contact us and provide details if you believe this document breaches copyrights.

We will remove access to the work immediately and investigate your claim. 
Green Open Access added to TU Delft Institutional Repository

'You share, we take care!' - Taverne project

https://www.openaccess.nl/en/you-share-we-take-care

Otherwise as indicated in the copyright section: the publisher is the copyright holder of this work and the author uses the Dutch legislation to make this work public. 


\title{
Asymmetric vortex shedding in the wake of an abruptly expanding annular jet
}

\author{
Maarten Vanierschot ${ }^{1}\left[\right.$ D $\cdot$ Mustafa Percin ${ }^{2} \cdot$ Bas W. van Oudheusden $^{3}$
}

Received: 16 July 2020 / Revised: 17 February 2021 / Accepted: 2 March 2021

(c) The Author(s), under exclusive licence to Springer-Verlag GmbH Germany, part of Springer Nature 2021

\begin{abstract}
In this paper, the structure of the turbulent wake behind the inner tube of a suddenly expanding annular jet flow is studied. The flow field is measured using tomographic particle image velocimetry and analyzed using proper orthogonal decomposition (POD). It was found that both the instantaneous and time-averaged central wakes behind the inner pipe are highly asymmetric despite the axisymmetric structure of the geometry. This asymmetry is the result of a bifurcation at low Reynolds numbers which persists up to the turbulent regime. The asymmetry induces a pair of counter-rotating vortices in the jet which are aligned with the main flow direction. Moreover, the asymmetry also induces a highly dynamical flow field. Analyzing the flow structures using POD shows that the wake oscillates around the asymmetric equilibrium position at a very low Strouhal number in the order of 0.01. On top of this motion, the inner shear layer oscillates with Strouhal numbers in the range of 0.1-0.3. This oscillation causes an asymmetric shedding of vortices of the hairpin type in the inner shear layer. As such, a local asymmetric region of very intensive mixing is induced near the stagnation point.
\end{abstract}

\section{Graphical Abstract}

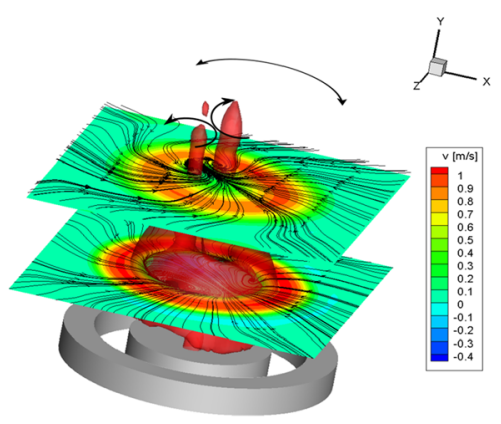

(a) Oscillation of the wake around the central axis (St is in the order of 0.01. The arrows indicatie the direction of rotation.

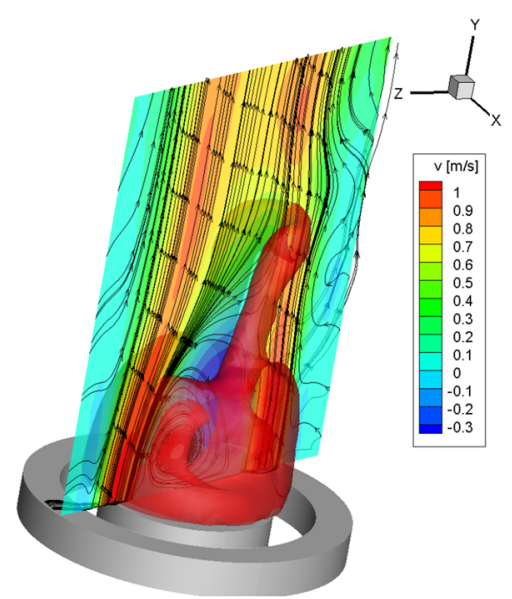

(b) Hairpin vortex shedding in the inner shear layer $(S t$ is in the order of 0.3 .

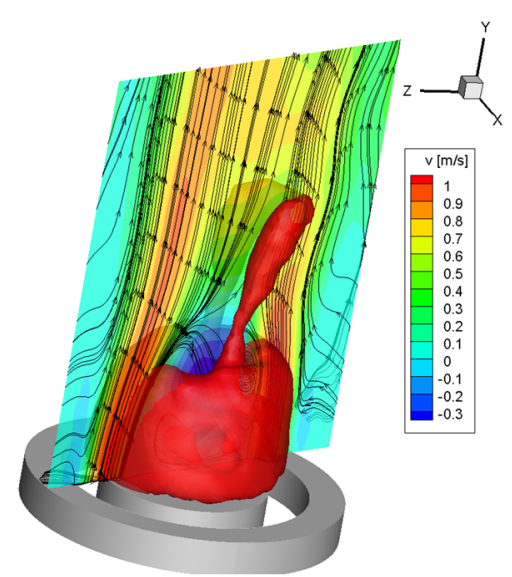

(c) Hairpin vortex shedding in the inner shear layer ( $S t$ is in the order of 0.13 .
Maarten Vanierschot

maarten.vanierschot@kuleuven.be

Extended author information available on the last page of the article

\section{Introduction}

Annular jets are encountered in many industrial applications involving bluff-body combustors, where they are applied in order to achieve a high degree of mixing, flue gas recirculation and flame stabilization (Beér and Chigier 1983; Gupta 
and Lilley 1984). The annular jet geometry is a very simple one with only two concentric tubes: one outer round tube with diameter $D_{o}$ and one closed inner one with diameter $D_{i}$, which acts as a bluff-body to the flow (figure 1). Moreover, this bluff-body can also be used to inject fuel in either cross-flow or co-flow mode (Dinesh and Kirkpatrick 2009; Vanierschot et al. 2009). Despite the simple geometry, the flow field is very complex. Two shear layers exist, one in the region between the jet and the environment, called the outer shear layer, and one in the region between the central recirculation zone (CRZ) behind the inner tube and the jet, called the inner shear layer (Dahm et al. 1992; Sadr and Klewicki 2003; Wawrzak et al. 2019). These shear layers interact with each other and their momentum thickness has a large influence on the dynamics of the CRZ (Wawrzak et al. 2019). This highly dynamical behavior of the CRZ originates from a nonlinear feedback mechanism of the position of the stagnation point back toward the nozzle tip, which alters its position, inducing large fluctuations in both space and time (Vanierschot and Van den Bulck 2011; Vanierschot et al. 2014).
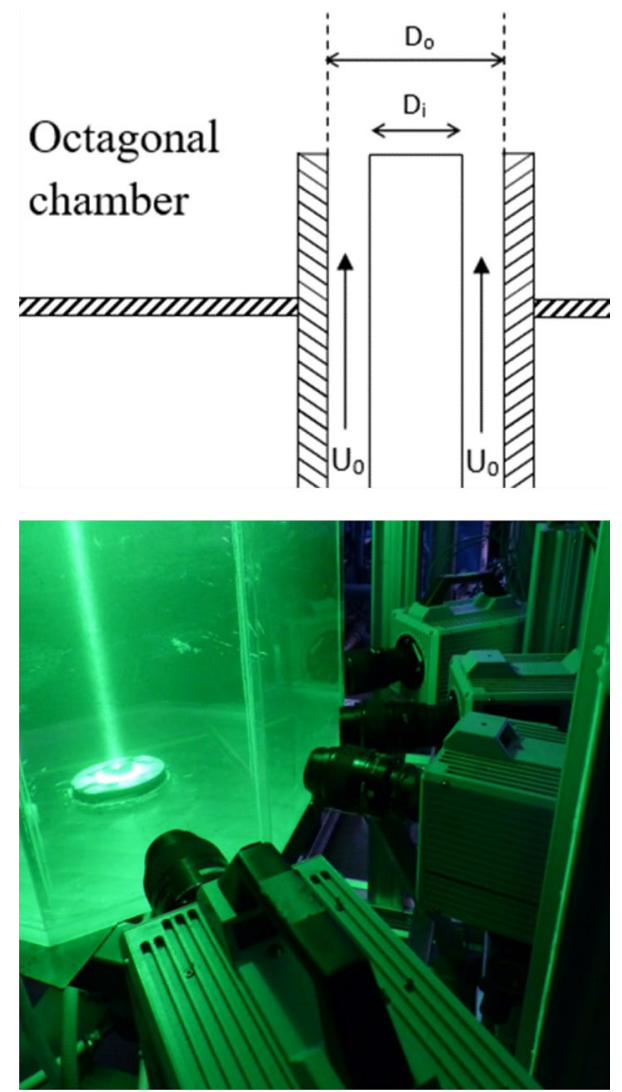

Fig. 1 Schematic view of the dimensions of the annular sudden expansion (top) and photograph of the tomographic PIV setup (bottom)
Although the annular jet geometry is axisymmetric, under some conditions the flow exhibits a spontaneous break in symmetry if the Reynolds number is above a certain threshold value, which is around 150 for the annular jet geometry used in this study (Vanierschot 2019). This break of symmetry has also been observed for similar axisymmetric bodies, like for instance spheres for Reynolds numbers above 250 (Fabre et al. 2008; Tiwari et al. 2019). The break of symmetry in annular jets was also confirmed by Del Taglia et al. (2009) who reported that for laminar, zero-swirl, incompressible annular jets, the break of symmetry is controlled by the Reynolds number and the blockage ratio which can be combined into a state parameter $\lambda$, defined as $\lambda=\ln R e+4.02 D_{i}^{2} / D_{o}^{2}$. At a certain critical value of the state parameter $\lambda_{c}$, the flow suddenly breaks symmetry (Del Taglia et al. 2004, 2009). This asymmetry also remains present in the turbulent regime at high Reynolds numbers (Vanierschot and Van den Bulck 2011; Ryzhenkov et al. 2019). The spatial aspects of the asymmetry can be studied using proper orthogonal decomposition (POD). POD is well capable of decomposing the velocity fluctuations into a part induced by the large-scale oscillation of the CRZ and a part attributed to turbulence as shown in the studies of Danlos et al. (2013) and PatteRouland et al. (2001). Reconstruction of the flow field using the two most energetic POD modes showed that mode 1 corresponds to the motion of the stagnation point, which is correlated with a significant asymmetry of the recirculation zone. However, since these 2D-PIV measurements were not time-resolved, no temporal information on this motion was available.

An annular jet can be seen as the limiting case of a coaxial jet with a velocity ratio (ratio between the velocity of the outer and the inner jet) of infinity. For coaxial jets in the laminar regime, Rehab et al. (1997) found that if the velocity ratio was higher than around 8 , an unsteady recirculation bubble appeared, which oscillates at a low frequency and high amplitude compared to the Kelvin-Helmholtz mode, which was also present. They reported a Strouhal number of $\mathrm{St}=f D_{i} / U_{o}=0.035$, where $D_{i}$ is the diameter of the inner jet and $U_{o}$ is the mean velocity of the outer jet. This frequency corresponds to the precession of the central bubble around the jet axis. Several other studies have reported similar frequencies (Segalini and Talamelli 2011; Buresti et al. 1994).

In view of this lack of temporal information, the main objective of the current study is to explore the dynamics of the three-dimensional flow structures in the CRZ via tomographic particle image velocimetry (tomographic-PIV). The experimentally obtained three- dimensional information allows to identify flow structures and dynamics which have not been reported previously in the literature. POD was used to extract the large-scale flow features, and it proved to be 
capable of separating the dynamic motion of the wake from the turbulent fluctuations in the flow field.

\section{Experimental setup and measurement procedure}

The experiments were conducted in a water tank facility at the Aerodynamic Laboratories of Delft University of Technology. The geometry of the annular jet is schematically shown in Fig. 1a. The pipe has an inner diameter $D_{i}=18$ $\mathrm{mm}$ and an outer diameter $D_{o}=27 \mathrm{~mm}$. The Reynolds number based on the mean axial velocity $\left(U_{o}=0.88 \mathrm{~m} / \mathrm{s}\right)$ and the hydraulic diameter of the annular jet $\left(D_{h}=9 \mathrm{~mm}\right)$ is 8000 . The symmetry axis of the jet is aligned with the (vertical) $y$-axis in the measurement coordinate system with the origin located at the annular pipe exit. The sudden expansion is located at the bottom wall of an octagonal water tank (600 $\mathrm{mm}$ of diameter and $800 \mathrm{~mm}$ of height), which is made of Plexiglass to enable full optical access for illumination and tomographic imaging (Percin and van Oudheusden 2015). The top of the tank has an overflow creating a constant recirculation of fluid with seeding particles in the system, which enables also seeding of the environment to avoid biased measurements in the shear layer.

Neutrally buoyant polyamide spherical particles of $56 \mu \mathrm{m}$ mean diameter were employed as tracer particles at a concentration of 0.65 particles $/ \mathrm{mm}^{3}$. The flow was illuminated by a double-pulsed Nd:YLF laser (Quantronix Darwin Duo, $2 \times 25 \mathrm{~mJ} /$ pulse at $1 \mathrm{kHz}$ ) at a wavelength of $527 \mathrm{~nm}$. The light scattered by the particles was recorded by four LaVision HighSpeedStar 6 CMOS cameras $(1024 \times 1024$ pixels, $5400 \mathrm{frames} / \mathrm{s}$, pixel pitch of $20 \mu \mathrm{m}$ ). The recording was done in a double-frame mode with a recording frequency of $50 \mathrm{~Hz}$, and a total of 2728 samples were collected, giving a total sampling time of 54.4 seconds. The sampling frequency was chosen such that the low-frequency dynamics of the wake could be captured, while still having a sufficient amount of periods measured. Based on the dimensions of the measurement volume and the average jet velocity, the measurements last for around 980 flow through times to ensure a complete dataset of instantaneous velocity fields for the POD analysis. Image pre-processing, volume calibration, self-calibration, reconstruction and three-dimensional cross-correlation-based interrogation were performed in LaVision DaVis 8.1.6, and the flow fields were analyzed using in-house developed software. The measurement volume was calibrated by scanning a calibration target through the measurement volume. A third-order polynomial was fitted as the mapping function that provides the relation between the image coordinates and the physical coordinates for each camera. The initial calibration was refined by means of the volume self-calibration technique (Wieneke 2008), resulting in a misalignment of less than 0.05 pixels. The raw images were pre-processed with background intensity removal and particle intensity normalization. The tomographic reconstruction was performed by using MLOS initialization (Atkinson and Soria 2009) and 10 CSMART iterations with Gaussian smoothing after each iteration. The particle images were then interrogated using an iterative, multigrid correlation with a window deformation procedure. Interrogation volumes of final size $48 \times 48 \times 48$ voxels with an overlap factor of $75 \%$ yielded a vector spacing of 0.56 $\mathrm{mm}$ in each direction. Spurious velocity vectors are removed by the universal median test, and a second-order polynomial regression in time and space was applied to reduce the noise in the resultant vector fields. A detailed analysis of the quality of the measurement data can be found in the work of Percin et al. (2017).

\section{Data processing and analysis}

The large scale flow structures obtained from the PIV measurements are extracted using proper orthogonal decomposition (POD) (Lumley 1970). In this study, we apply the snapshot POD variant proposed by Sirovich (1987), including all velocity components. The velocity field is decomposed into a set of spatial modes $\boldsymbol{\Phi}_{i}(\boldsymbol{x})$ and temporal coefficients $a_{i}(t)$ as

$\boldsymbol{v}(\boldsymbol{x}, t)=\overline{\boldsymbol{v}}(\boldsymbol{x})+\boldsymbol{v}^{\dagger}(\boldsymbol{x})=\overline{\boldsymbol{v}}(\boldsymbol{x})+\sum_{i=1}^{N} a_{i}(t) \boldsymbol{\Phi}_{i}(\boldsymbol{x})$,

where $v$ is a velocity vector with components $u, v$ and $w$. The overbar denotes time-averaged quantities, and hence, only the fluctuating velocity field is decomposed. For the $N$ snapshots in time obtained by Tomo-PIV, the temporal coefficients $\boldsymbol{a}_{i}=\left[a_{i}\left(t_{1}\right), a_{i}\left(t_{2}\right), \ldots, a_{i}\left(t_{N}\right)\right]^{T}$ and mode energies $\lambda_{i}$ can be obtained by solving the eigenvalue problem

$\boldsymbol{R} \boldsymbol{a}_{i}=\lambda_{i} \boldsymbol{a}_{i} ; \lambda_{1} \geq \lambda_{2} \geq \cdots \geq \lambda_{n} \geq 0$,

where the elements of the correlation matrix $\boldsymbol{R}$ are given by

$R_{i, j}=\frac{1}{N}\left\langle v^{\dagger}\left(x, t_{i}\right), v^{\dagger}\left(x, t_{j}\right)\right\rangle$.

As spatial inner product $\langle$,$\rangle , the L^{2}$ norm is usually taken. The spatial modes are obtained by the projection of the fluctuating velocity fields onto the temporal coefficients as 

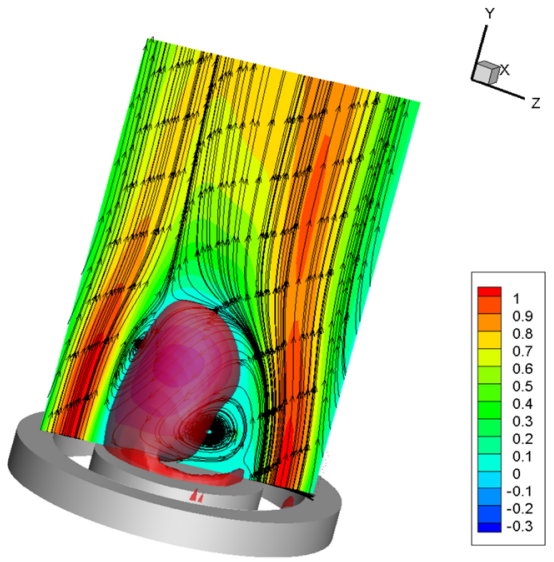

(a) In-plane axial velocity ( $V_{x}$ component) contours.

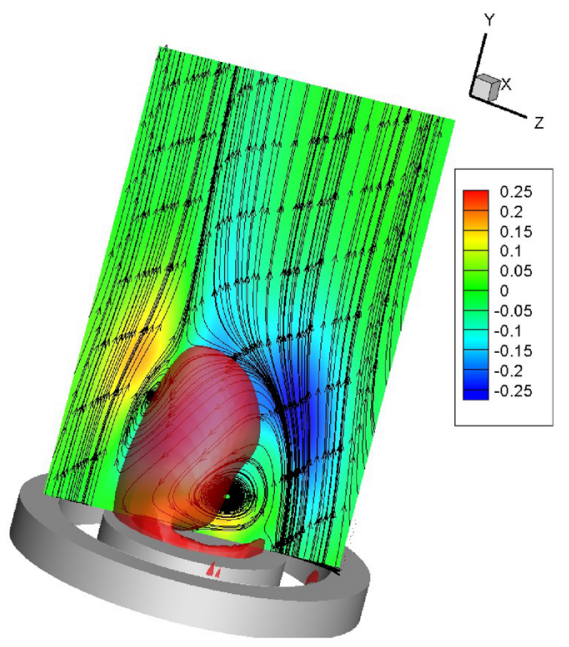

(c) In-plane radial velocity $\left(V_{r}\right.$ component) contours.

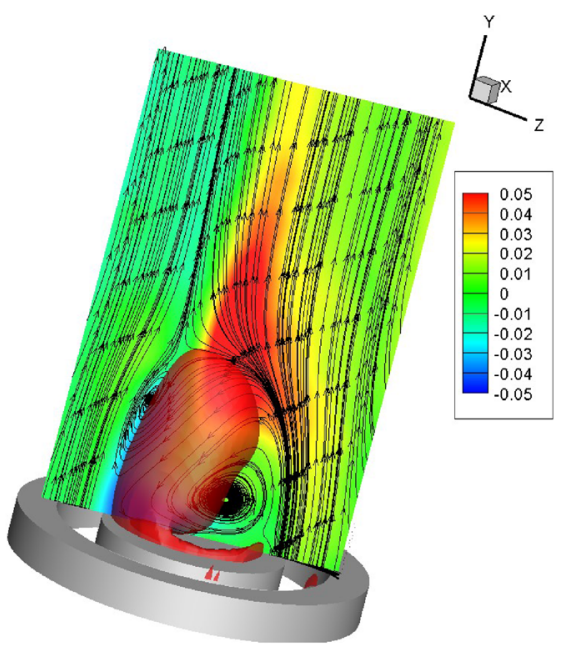

(e) Out-of-plane velocity ( $V_{t}$ component) contours.

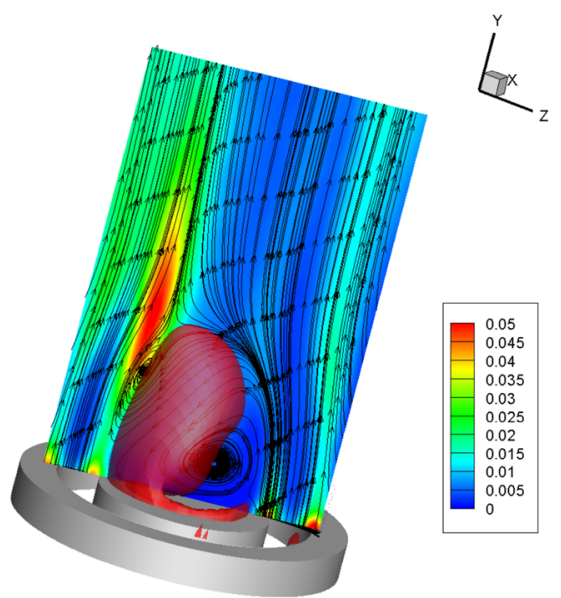

(b) In-plane fluctuating axial velocity $\left(\overline{v_{x}^{\prime} v_{x}^{\prime}}\right)$ contours.

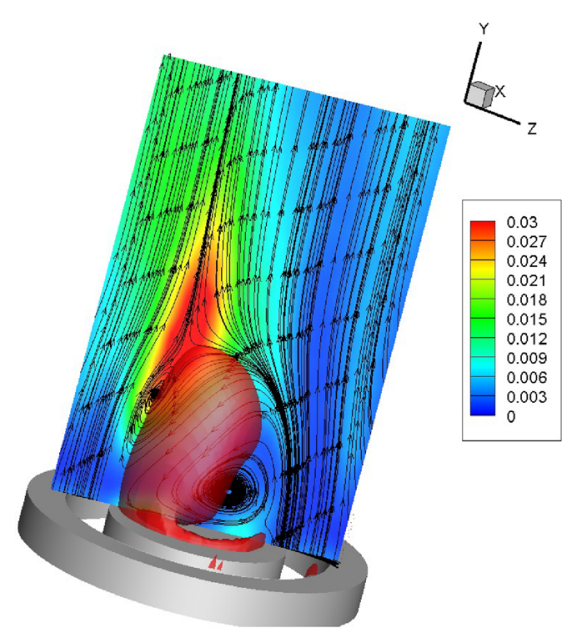

(d) In-plane fluctuating radial velocity $\left(\overline{v_{r}^{\prime} v_{r}^{\prime}}\right)$ contours.

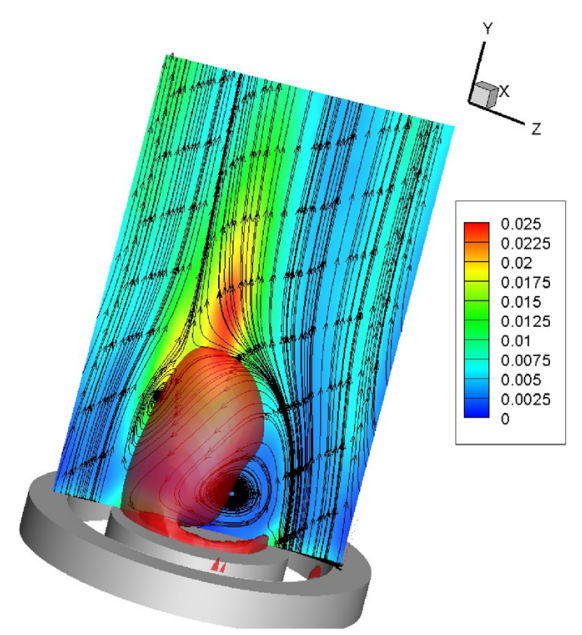

(f) Out-of-plane fluctuating velocity $\left(\overline{v_{t}^{\prime} v_{t}^{\prime}}\right)$ contours. 
4Fig. 2 Velocity contours of the time-averaged flow field in the planeof-asymmetry of the flow. The recirculation zone is depicted by the red isosurface of zero axial velocity. The in-plane streamlines clearly reveal the asymmetry of the wake in this cross-sectional plane

$\boldsymbol{\Phi}_{i}(\boldsymbol{x})=\frac{1}{N \lambda_{i}} \sum_{j=1}^{N} a_{i}\left(t_{j}\right) \boldsymbol{v}^{\dagger}\left(\boldsymbol{x}, t_{j}\right)$

The flow field can be reconstructed as in Eq. 1 using a number of selected modes.

An important aspect in the flow field analysis and decomposition using POD is the identification of linked modes. For instance, the dynamical behavior of periodic coherent structures is usually described by a mode pair. A mode pair is defined as two modes in the decomposition which have the same spectral content and a constant phase difference of $\pm \pi / 2$. As such, reconstructing the flow field with a mode pair describes the precession of a coherent structure in the flow. A linked mode pair $a_{i}(t)$ and $a_{j}(t)$ can be identified by looking at the harmonic correlation of the eigenvectors of the DMD decomposition of the temporal coefficients. More detailed information on the identification of mode pairs can be found in the work of Sieber et al. (2016).

\section{Results and discussion}

\subsection{Statistical flow field characterization}

The time-averaged velocity fields are shown in Figs. 2 and 3. Despite the axisymmetric geometry of the experimental setup (both tubes were aligned by an in-house designed positioning mechanism, making it possible to center them within an accuracy of $0.004 D_{o}$. (Vanierschot and Van den Bulck 2011)), the wake behind the inner pipe is asymmetric. Analysis of the 3D structure shows the existence of one stationary plane-of-asymmetry aligned with the flow direction (Fig. 2), in which the wake is asymmetric and one plane-of-symmetry (Fig. 3), in which the wake is symmetric. Both planes are perpendicular to each other. This axisymmetric nature is the result of a bifurcation at a threshold Reynolds number of 150 for the current geometry (Vanierschot 2019). This induces a 'locking' of the flow field toward an asymmetric state. Instantaneous asymmetric wakes were also reported for spheres and axisymmetric afterbodies, but there is no preferential direction of locking if the setup is well alligned with the flow (Grandemange et al. 2012, 2014; Gentile et al. 2016). However, a slight pitch of the axisymmetric body can make the wake asymmetric and locking it in a fixed position. This is also confirmed in this study as for a given alignment of the inner tube, the position of asymmetry is constant even if the flow is switched off and on. Hence, in this experimental study, the locking is induced by slightly asymmetric conditions of the setup, while numerical studies confirm locking of the flow to the grid topology (Del Taglia et al. 2009; Ryzhenkov et al. 2019; Ogus et al. 2016). With respect to both planes, the velocity vector is decomposed into three components: two inside the plane: a component in the flow direction $\left(V_{x}\right.$, Figs. 2a and 3a) and one component perpendicular to the flow direction $\left(V_{r}\right.$, Figs. $2 \mathrm{c}$ and $3 \mathrm{c})$ and a velocity component perpendicular to the plane $\left(V_{t}\right.$, Figs. 2e and 3e). The normal Reynolds stresses of these velocity components, $\left(\overline{v_{x}^{\prime} v_{x}^{\prime}}, \overline{v_{r}^{\prime} v_{r}^{\prime}}\right.$ and $\left.\overline{v_{t}^{\prime} v_{t}^{\prime}}\right)$ are shown in Figs. 2b, d, f and 3b, d, f. Large values for all stresses can be found in the inner and outer shear layer of the jet, with the highest values for the radial and azimuthal stresses in a very local region near the stagnation point of the wake (see Fig. 2b). Several authors reported high values in this region which are attributed to the motion of the stagnation point (Patte-Rouland et al. 2001; Danlos et al. 2013; Grandemange et al. 2012) and depict the highly dynamical behavior of the CRZ. Comparison with the same jet geometry at a Reynolds number of 180 shows a remarkable similarity between both profiles of Reynolds stresses (Ogus et al. 2016). This similarity indicates a large contribution to the second-order statistics of coherent flow structures with length scales much larger than the turbulent eddies.

Figure 4 reveals the structure of the flow downstream of the stagnation point of the central wake. The location of the middle measurement plane corresponds to the farthest location of the stagnation surface in figures 2 and 3. Vortex identification based on the Q-criterion (Jeong and Hussain 1995) shows the existence of a pair of counter-rotating vortices which is aligned with the main flow direction. These vortices are also identified by the in-plane streamlines shown at various cross-sectional planes in the flow. They originate from the envelope of the asymmetric central recirculation zone near the stagnation point due to the inward motion of the jet. These features have also been observed for laminar flow conditions (Ogus et al. 2016), but this study is the first one to identify them occurring in turbulent flow as well. A closer look at the vorticity vector, i.e., $\omega=\boldsymbol{\nabla} \times \boldsymbol{v}$, in Fig. 5 shows that the amplitude of the axial vorticity is maximal in the center of each vortex of the pair and that the most dominant component is in the y-direction. This shows that the rotation is mainly in that direction and the sense of the 


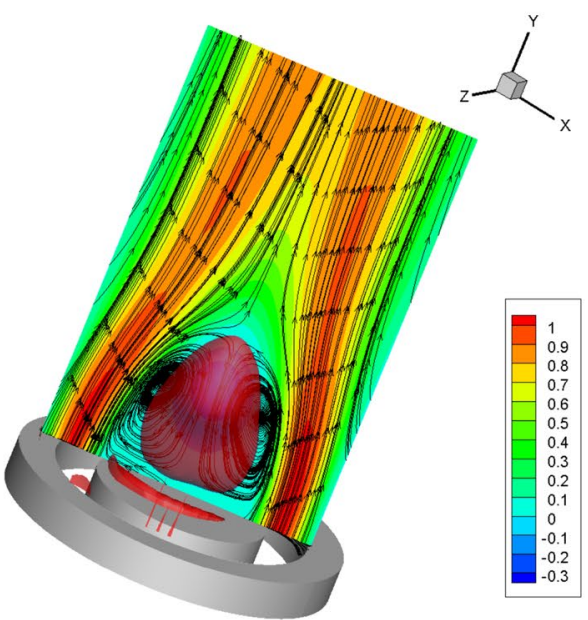

(a) In-plane axial velocity ( $V_{x}$ component) contours.

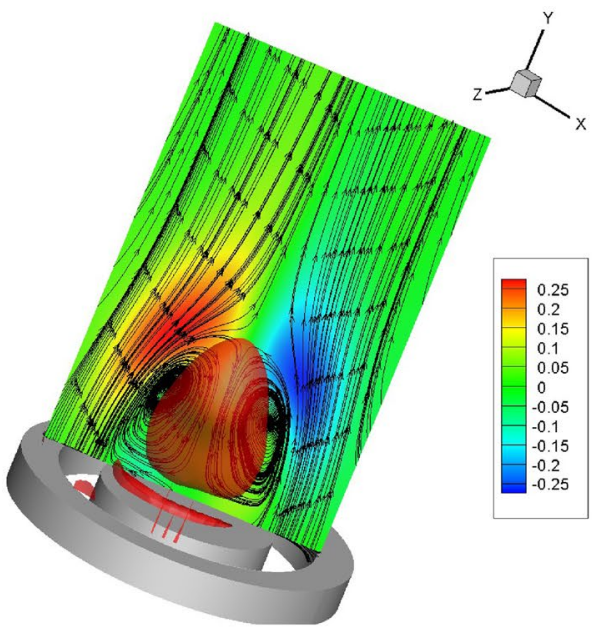

(c) In-plane radial velocity ( $V_{r}$ component) contours.

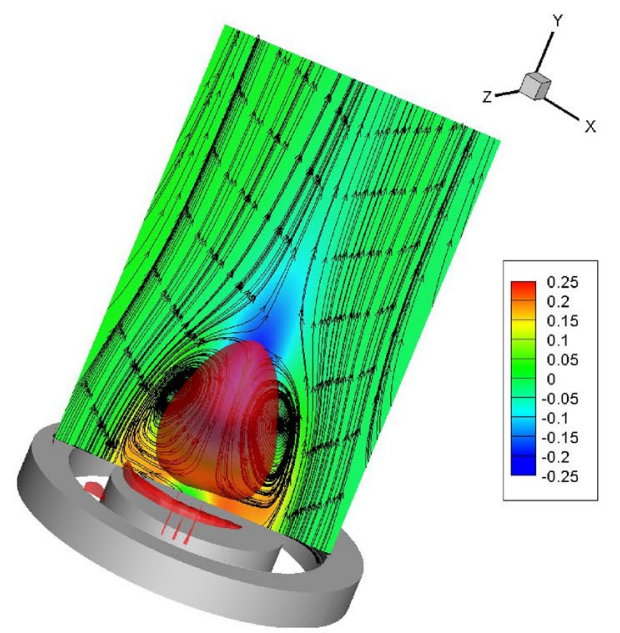

(e) Out-of-plane velocity ( $V_{t}$ component) contours.

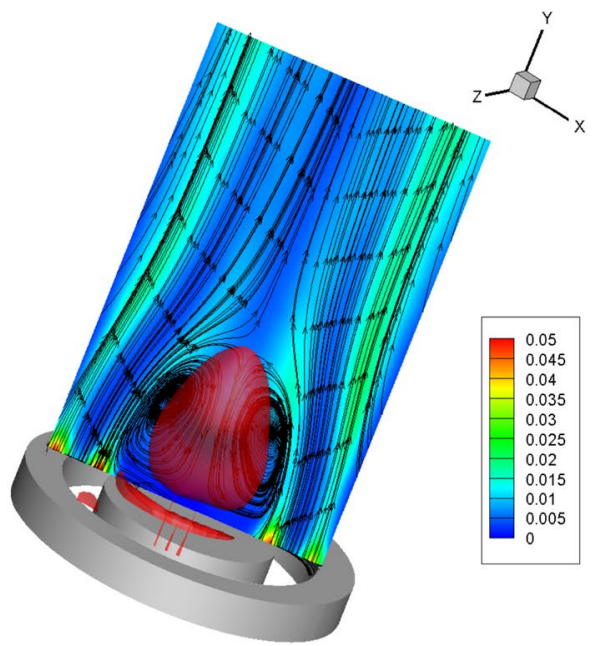

(b) In-plane fluctuating axial velocity $\left(\overline{v_{x}^{\prime} v_{x}^{\prime}}\right)$ contours.

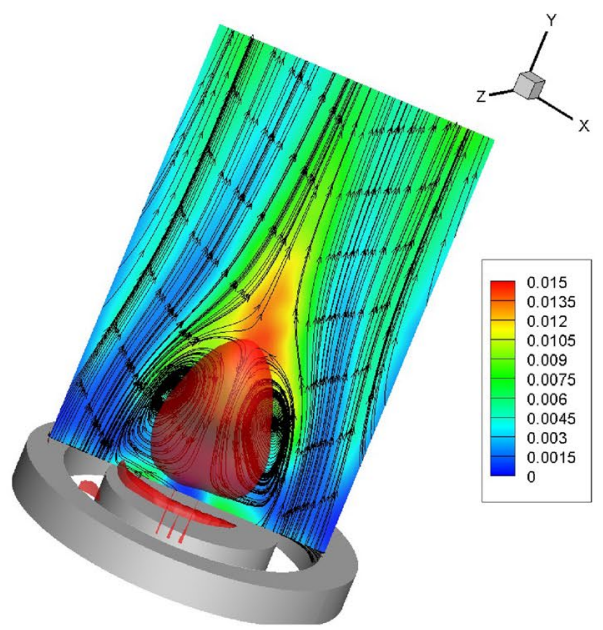

(d) In-plane fluctuating radial velocity $\left(\overline{v_{r}^{\prime} v_{r}^{\prime}}\right)$ contours.

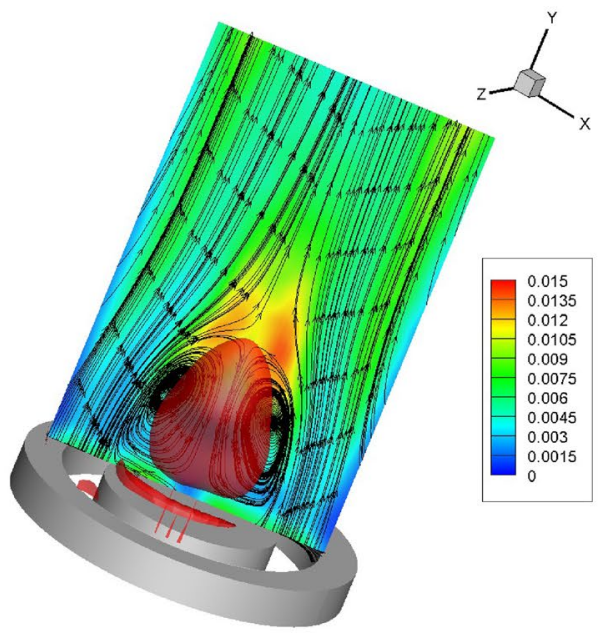

(f) Out-of-plane fluctuating velocity $\left(\overline{v_{t}^{\prime} v_{t}^{\prime}}\right)$ contours. 
4Fig. 3 Velocity contours of the time-averaged flow in the plane-ofsymmetry of the flow. The recirculation zone is depicted by the red isosurface of zero axial velocity. The in-plane streamlines clearly reveal the symmetry of the wake in this cross-sectional plane

vectors also shows a counter-rotating pair. However, the vorticity vector is not completely aligned with the y-axis, meaning there is still some rotation in the other directions. These other components get larger when moving away from the vortex centers indicating the spiraling motion of flow near the vortex pair.

\subsection{Flow field dynamics}

To study the dynamical features of the flow, the velocity fields were decomposed using POD (Sirovich 1987). Analysis of the spectral content of the time coefficients, shown in Fig. 6, reveals the existence of several dynamic structures in the flow field with specific frequency ranges. These structures can be categorized in three types based on the Strouhal number (defined on the basis of the diameter of the central bluff-body and mean axial velocity of the jet) at which the peak in the frequency spectrum is situated: structures which have a peak around $S t=0.01$ (e.g., mode 1), 0.3 (e.g., modes 2-5) or around 0.13 (e.g., modes 6-7), see Fig. 7. Each distinct frequency range (except for mode one) involves multiple POD modes which have the same amplitude and are arranged in mode pairs according to their harmonic correlation and the examples given in Fig. 7 are the ones with the lowest mode number. Reconstructing the velocity fields using the mean flow field and the specific mode pair can be represented by a reconstruction with the maximal and minimal time coefficient of one mode of the pair as the other one is zero since the phase shift between both coefficients is $\pi / 2$. The reconstruction for the first type of dynamic structures (where $S t$ is in the order of 0.01) is shown in Fig. 8. This mode describes the rotation of the wake around the central axis of the jet. This motion is not a precession, but an oscillation around the asymmetric equilibrium position (shown in Fig. 4) with an azimuthal amplitude of $25^{\circ}$. This oscillation induces an asymmetry in the strength of each vortex of the pair. On a time-averaged basis, the strength of both vortices is equal, but instantaneously at maximal rotation angle (Fig. 8a), one vortex is stronger compared to the other one. In the minimal position of the oscillation, this is reversed (Fig. 8b). The study of Vanierschot et al. (2014) reported similar structures and associated frequencies and the stereoscopic PIV measurements also showed the lack of a precession. However, the study of Rehab et al. (1997) for a coaxial jet of infinite velocity ratio reported a higher Strouhal number of 0.035 and showed that the recirculation zone precesses around the central axis. This mechanism was explained as the result of a coupled perturbation of the inner shear layer by recirculation from the stagnation point. Most likely the difference with this study is caused by the absence of a closed inner tube in the latter one, changing the structure of the inner shear layer perturbation.

The reconstructed velocity fields (using the mean flow field and the specific mode pair consisting of modes 2 and 3 ) for the second type of dynamic structures ( $S t$ is in the order of 0.3 ) are shown in Fig. 9. The velocity field is reconstructed using the maximum and minimal temporal coefficient of mode 2 as mode three is $\pi / 2$ radians out of phase, and hence, the corresponding coefficient is zero at those time instants. This mode pair describes the formation and axial convection of hairpin vortices in the inner shear layer. This asymmetric vortex shedding can also be found in the laminar regime and is a result of the asymmetric wake and associated shear layer (Ogus et al. 2016). Moreover, the shedding frequencies of the laminar and turbulent flows are equal within measurement accuracy and are similar to typical values for bluff-body wakes of similar axisymmetric objects like spheres and discs (Fabre et al. 2008; Roshko 1954; Dousset and Pothrat 2010; Tiwari et al. 2019; Tomboulides and Orszag 2000; Robertson et al. 2015). However, in the before mentioned papers, these frequencies are associated with Kelvin-Helmholtz instabilities of the shear layer. They can exist next to the hairpin vortex shedding and start for a sphere for instance at a Reynolds number of 800 (Tiwari et al. 2019). This study shows that the shear layer rollup also results in the formation of hairpin vortices.

The reconstructed velocity fields (using the mean flow field and the specific mode pair) for the third type of dynamical structures (where $S t$ is in the order of 0.13) are shown in Fig. 10. Similar like the structures at $S t=0.3$, the velocity field is reconstructed using only the maximum and minimal temporal coefficient of the lowest mode number, which is mode 6 in this case. Similar to the second type, this mode also describes the formation and convection of hairpin vortices in the inner shear layer. Note that the hairpin vortex in Fig. 10b is about to form as it is located very close to the stagnation point's position. This hairpin shedding can also be found in other bluff-body flows like in spheres (Tiwari et al. 2019) or square cylinders (Dousset and Pothrat 2010) and is attributed to von Karman shedding in the wake flow. This coincides with the high fluctuating velocity region in Fig. $2 b$ and as such, the hairpin vortices have a large contribution to the asymmetric profile of the fluctuating velocity near the stagnation point. Giving the same spatial structure 


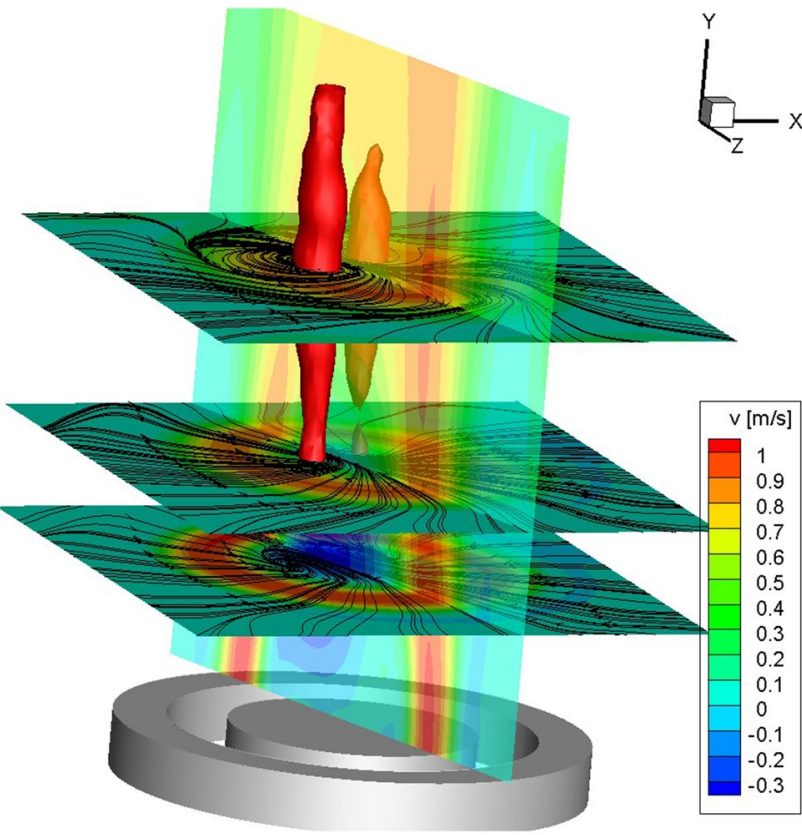

Fig. 4 Time-averaged asymmetric equilibrium position of the wake. The counter-rotating vortex pair is visualized by the isocontour of Q $=0.001$. The in-plane streamlines at several cross-sectional planes show the counter-rotating motion of the vortex pair, while the arrows in the figure indicate the rotational direction of the vortex pair

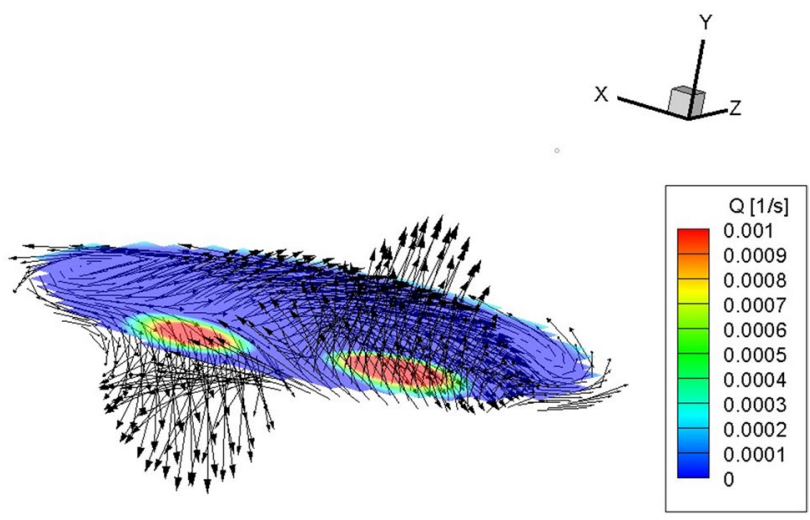

Fig. 5 Vorticity vectors in the upper measurement plane shown in figure 4. The contours show values of $\mathrm{Q}$ and the color levels are cut to visualize the center of the vortex pair

and the almost double frequency, one might think that the second type of structures is second harmonics of the third type. However, the scatter plots of the temporal coefficients of the two modes (not shown here) do not show the typical eight shape as what would be expected from Lissajous figures for second harmonics. Nevertheless, plotting the phase

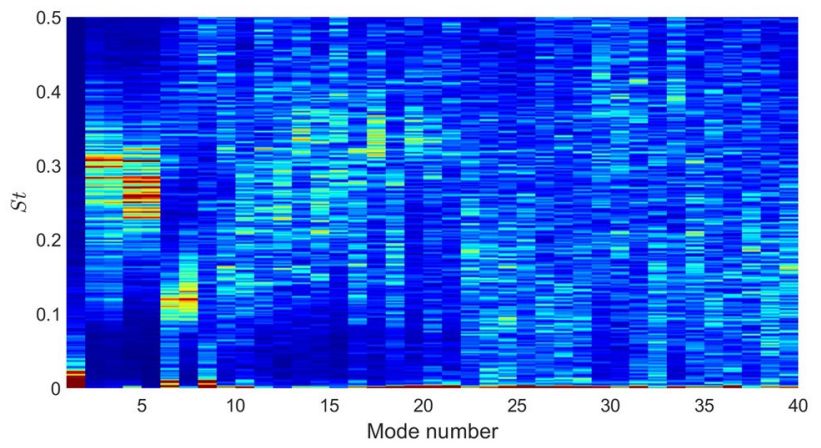

(a) Normalised frequency spectrum of the temporal coefficients of the first 40 POD modes.

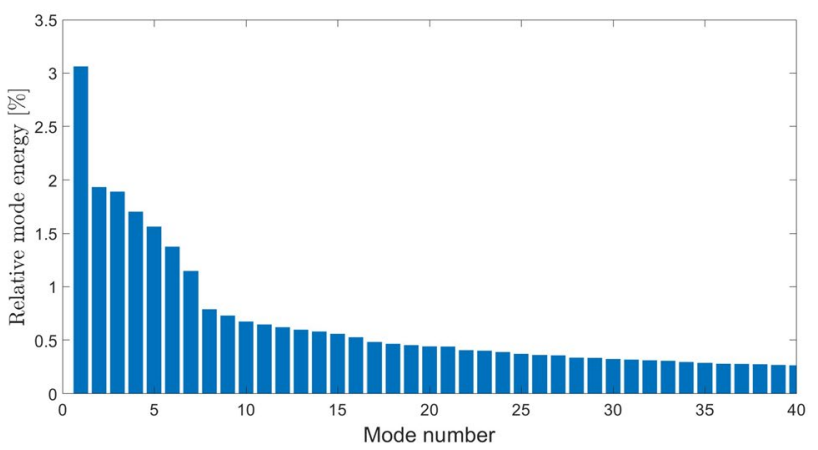

(b) Relative energy contribution of the first 40 POD modes.

Fig. 6 Characteristics of the first 40 POD modes

portrait might be corrupted by intermittency and phase distortion, and therefore, a more detailed statistical analysis is given in Fig. 11. If mode 2 would be a second harmonic of mode 7 , the statistical histogram of the variable $2 \phi_{7}-\phi_{2}$, where $\phi$ is denoted as the phase angle of the time coefficient, should give one clear peak. Figure 11 shows that there is no statistical correlation and hence it can be concluded that both modes are not harmonics of each other. This confirms that the origin is a different physical mechanism, i.e., KelvinHelmholtz instabilities for structures with a Strouhal number around 0.3 and von Karman shedding for the structures with Strouhal number around 0.13 .

\section{Conclusions}

This paper studies the structures in the wake of an annular jet geometry, representative of a bluff-body combustor. It was found that the time-average flow field was asymmetric, resulting from a bifurcation at lower Reynolds number. This asymmetric state induces a counter-rotating 


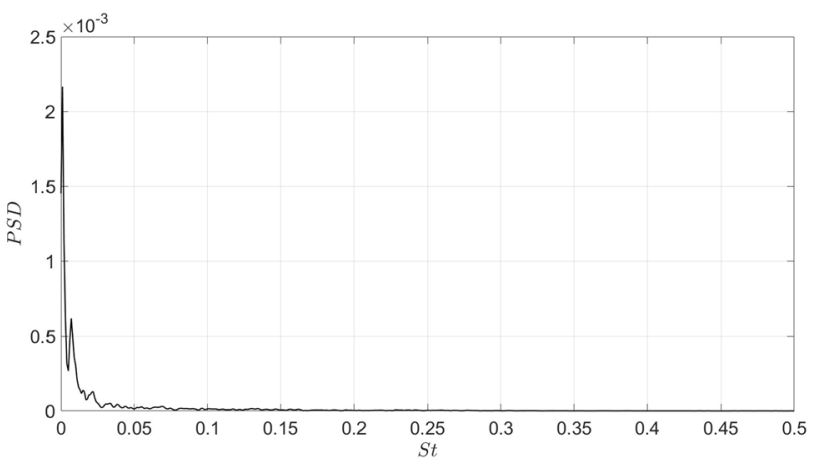

(a) Mode one

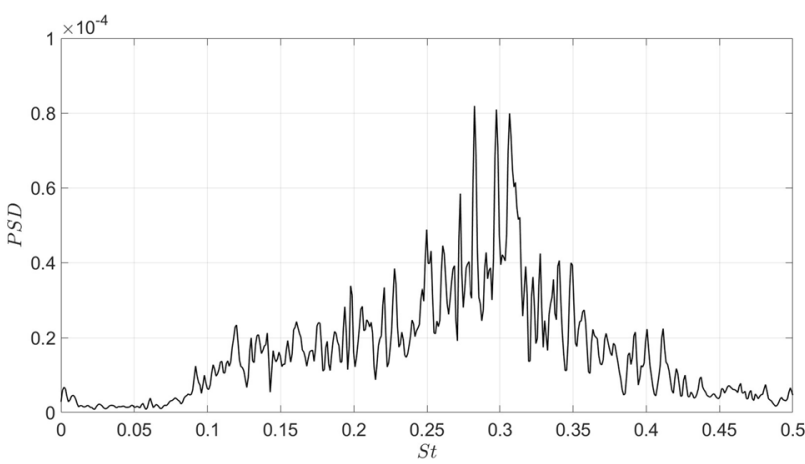

(b) Mode two

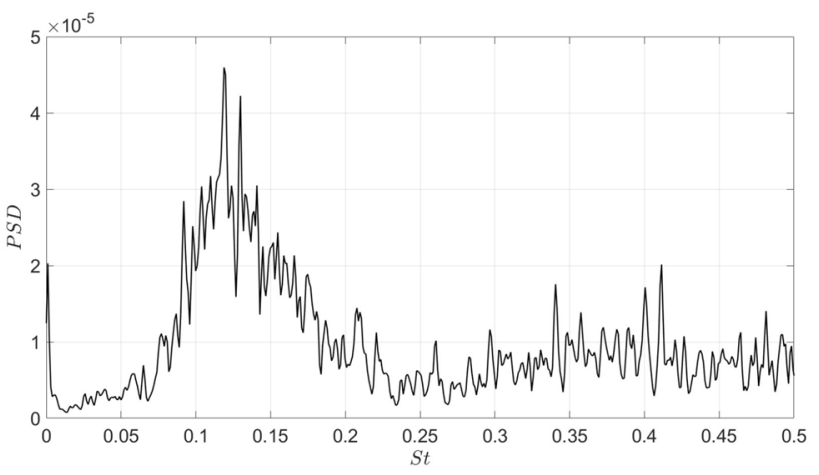

(c) Mode seven

Fig. 7 Spectral content of the time coefficients of selected POD modes as representative for the three specific frequency intervals

vortex pair which is aligned with the main flow direction. Analysis of the dynamic structures in the wake shows that these can be categorized in three types dependent on their frequency peak in the spectrum: type one which are modes with a Strouhal number in the order of 0.01 , representing the oscillation of the wake around the central axis, type

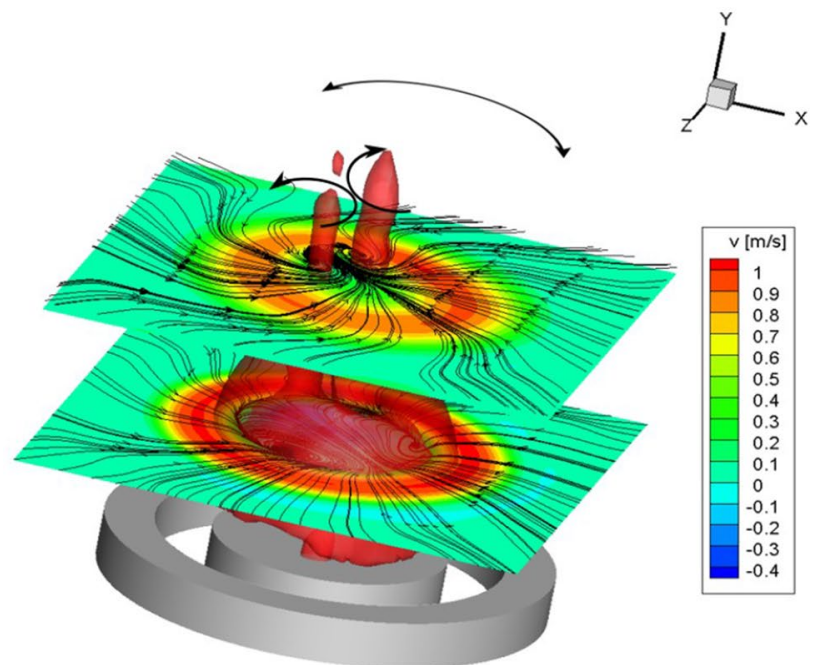

(a) Reconstructed velocity field using the maximal time coefficient of mode 1 of the pair.

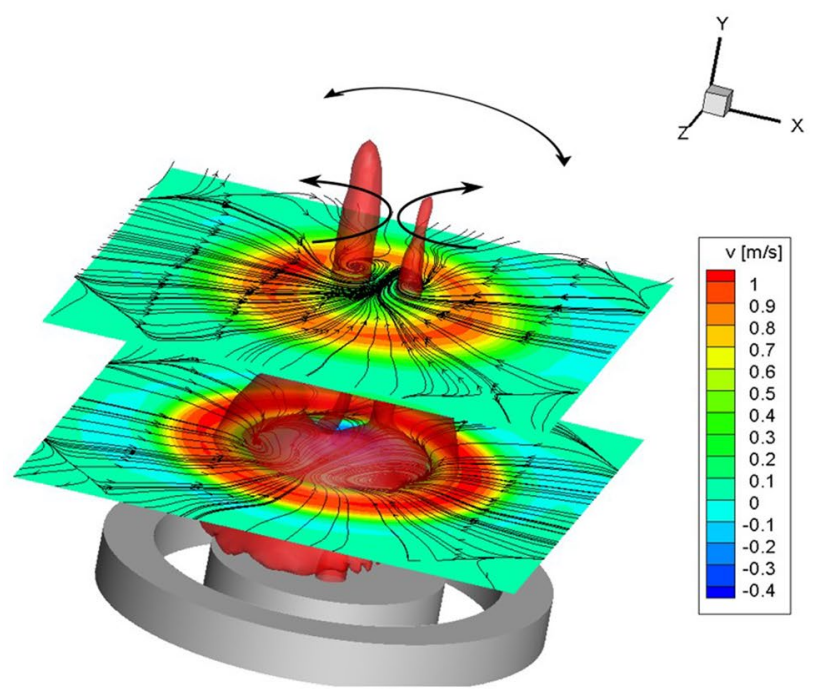

(b) Reconstructed velocity field using the minimal time coefficient of mode 1 of the pair.

Fig. 8 Reconstructed velocity fields using mode 1 . The red surfaces are isosurfaces of $\mathrm{Q}=0.001$. The big arrow denotes the oscillating motion of the wake, while the small arrows indicate the direction of rotation of the counter-rotating vortex pair

two containing modes having a $S t$ in the order of 0.13 and type three containing modes with $S t$ in the order of 0.3 . The latter ones describe the shedding of hairpin vortices in the inner shear layer. These vortices originate near the stagnation point, and as such, the hairpin vortices have a large contribution to the asymmetry of the high fluctuating velocity region near the stagnation point. 


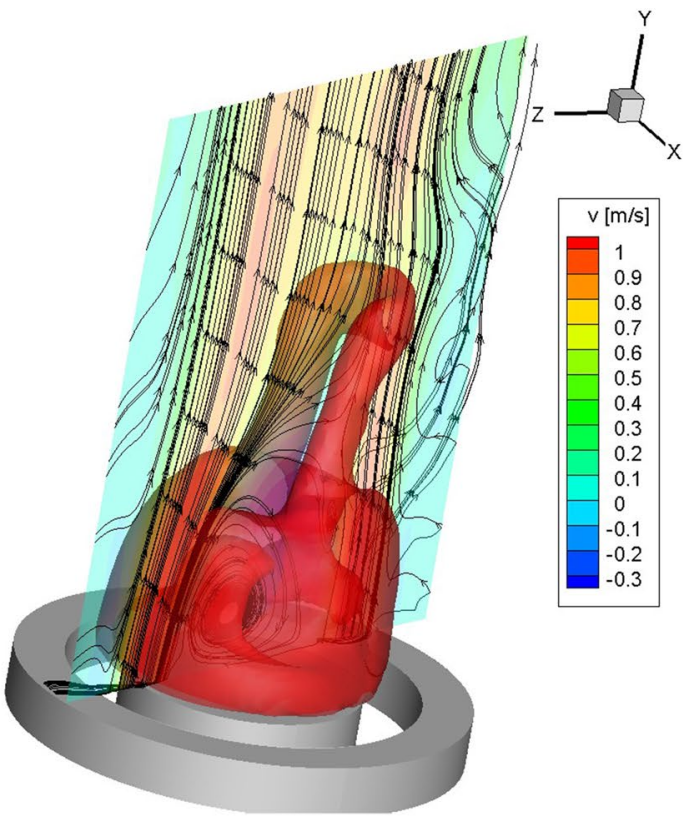

(a) Reconstructed velocity field using the maximal time coefficient of mode 2 of the pair.

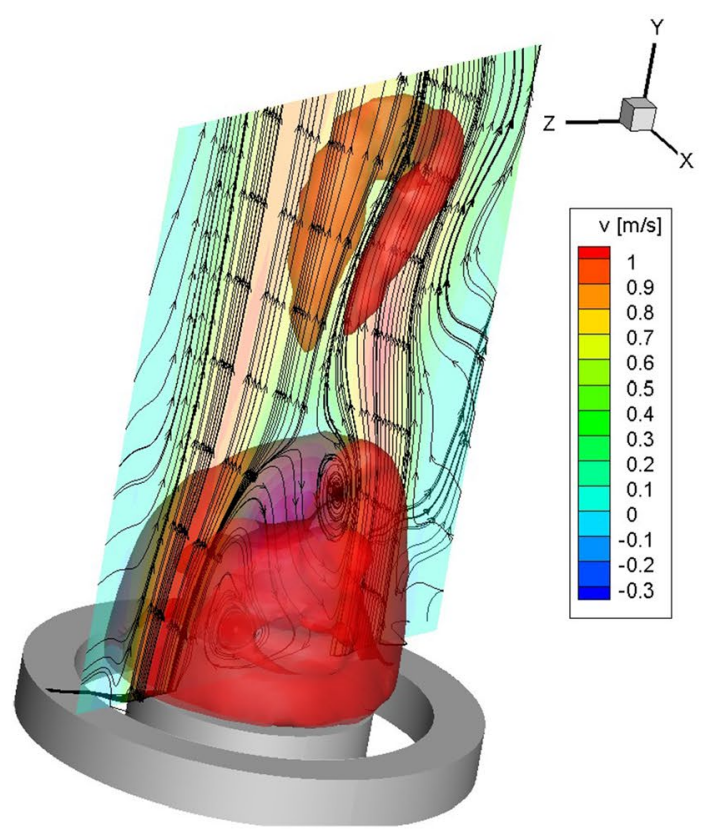

(b) Reconstructed velocity field using the minimal time coefficient of mode 2 of the pair.

Fig. 9 Reconstructed velocity fields using mode 2. The red surfaces are isosurfaces of $Q=0.001$, which visualizes the hairpin vortex shed from the inner shear layer

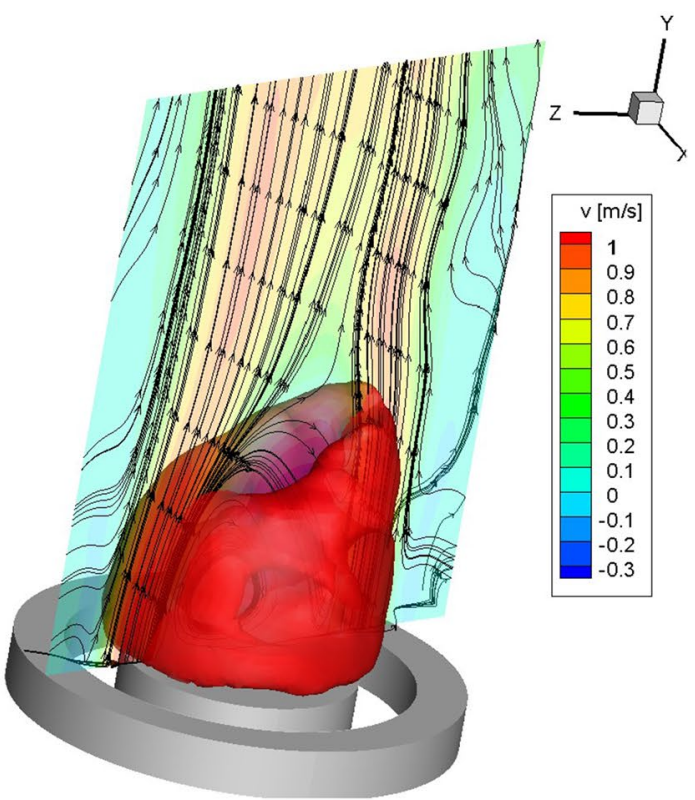

(a) Reconstructed velocity field using the maximal time coefficient of mode 7 of the pair.

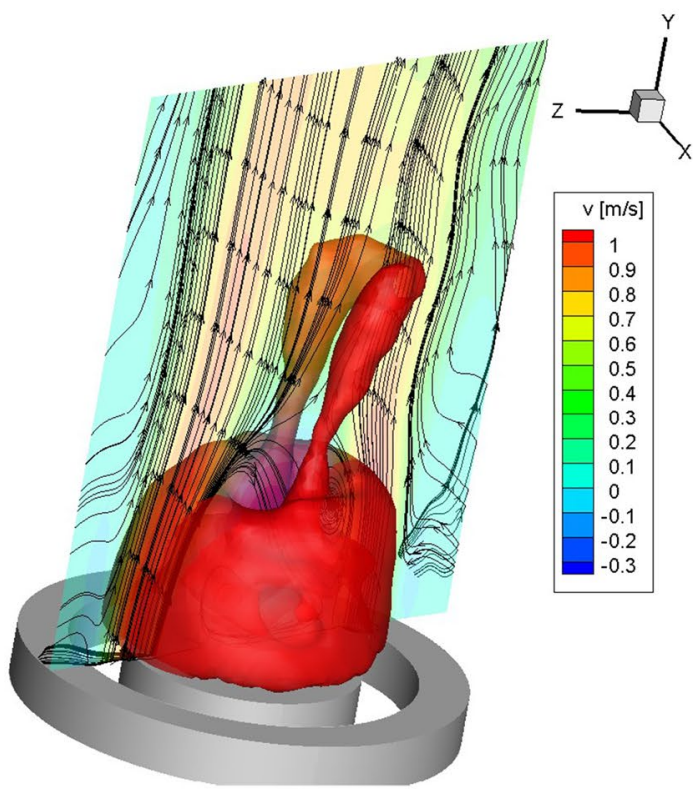

(b) Reconstructed velocity field using the minimal time coefficient of mode 7 of the pair.

Fig. 10 Reconstructed velocity fields using mode 7. The red surfaces are isosurfaces of $Q=0.002$, which visualizes the hairpin vortex shed from the inner shear layer 


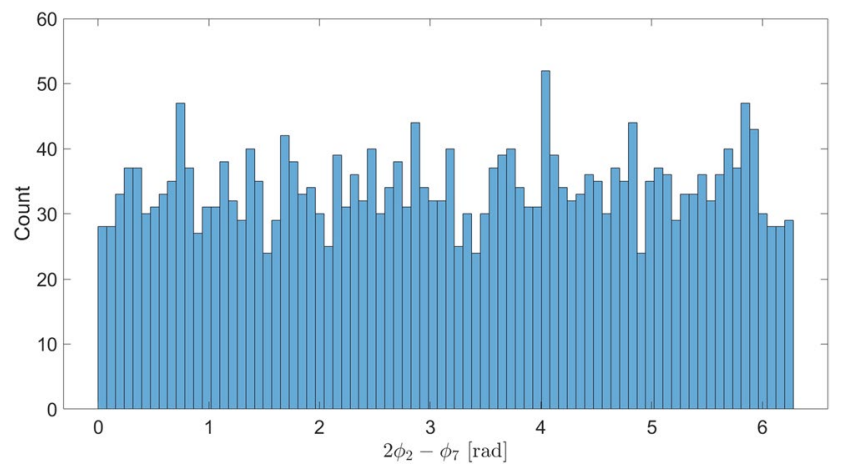

Fig. 11 Histogram of the phase difference of modes 2 and 7, i.e., $2 \phi_{7}-\phi_{2}$

Acknowledgements The corresponding author wishes to thank the FWO Flanders and the J.M. Burgerscentrum for fluid mechanics for financially supporting his stay to perform the measurements at the water tank facility at Delft University of Technology (TU Delft).

\section{References}

Atkinson C, Soria J (2009) An efficient simultaneous reconstruction technique for tomographic particle image velocimetry. Exp Fluids 47:553

Beér J, Chigier N (1983) Combustion Aerodynamics. Krieger Pub Co Buresti G, Talamelli A, Petagna P (1994) Experimental characterization of the velocity field of a coaxial jet configuration. Exp Therm Fl Sc 9:135-146

Dahm W, Frieler C, Tryggvason G (1992) Vortex structure and dynamics in the near field of a coaxial jet. J Fluid Mech 241:371-402

Danlos A, Lalizel G, Patte-Rouland B (2013) Experimental characterization of the initial zone of an annular jet with a very large diameter ratio. Exp Fluids 54:1418

Del Taglia C, Blum L, Gass J, Ventikos Y, Poulikakos D (2004) Numerical and experimental investigation of an annular jet flow with large blockage. J Fluid Eng - Trans ASME 126:375-384

Del Taglia C, Moser A, Blum L (2009) Spontaneous break of symmetry in unconfined laminar annular jets. J Fluid Eng - Trans ASME 131:081202

Dinesh KR, Kirkpatrick M (2009) Study of jet precession, recirculation and vortex breakdown in turbulent swirling jets using les. Comput Fluids 38:1232-1242

Dousset V, Pothrat A (2010) Formation mechanism of hairpin vortices in the wake of a truncated square cylinder in a duct. J Fluid Mech 653:519-536

Fabre D, Auguste F, Magnaudet J (2008) Bifurcations and symmetry breaking in the wake of axisymmetric bodies. Phys Fluids 20:051702

Gentile V, Schrijer F, Oudheusden BV, Scarano F (2016) Low-frequency behavior of the turbulent axisymmetric near-wake. Phys Fluids 28:065102

Grandemange M, Gohlke M, Parezanovi V, Cadot O (2012) On experimental sensitivity analysis of the turbulent wake from an axisymmetric blunt trailing edge. Phys Fluids 24:035106

Grandemange M, Gohlke M, Cadot O (2014) Statistical axisymmetry of the turbulent sphere wake. Exp Fluids 55:1838
Gupta A, Lilley G (1984) Swirl Flows. Abacus, Kent

Jeong J, Hussain F (1995) On the identification of a vortex. J Fluid Mech 285:69-94

Lumley J (1970) Stochastic tools in turbulence. Applied mathematics and mechanics series. Academic press, New York

Ogus G, Baelmans T, Vanierschot M (2016) On the flow structures and hysteresis of laminar swirling jets. Phys Fluids 28:123604

Patte-Rouland B, Lalizel G, Moreau J, Rouland E (2001) Flow analysis of an annular jet by particle image velocimetry and proper orthogonal decomposition. Meas Sci Technol 12:1404-1412

Percin M, van Oudheusden B (2015) Three-dimensional flow structures and unsteady forces on pitching and surging revolving flat plates. Exp Fluids 56:1-19

Percin M, Vanierschot M, van Oudheusden B (2017) Analysis of the pressure fields in a swirling annular jet flow. Exp Fluids 58:1-13

Rehab H, Villermaux E, Hopfinger E (1997) Flow regimes of large velocity-ratio coaxial jets. J Fluid Mech 345:357-381

Robertson E, Choudhury V, Bhushan S, Walters DK (2015) Validation of openfoam numerical methods and turbulence models for incompressible bluff body flows. Comput Fluids 123:122-145

Roshko A (1954) On the drag and shedding frequency of two-dimensional bluff bodies. Tech Rep 3169 NACA Tech Notes

Ryzhenkov V, Abdurakipov S, Mullyadzhanov R (2019) The asymmetry of the recirculation zone of the annular jet with different diameter ratio. J Phys Conf Series 1382:012035

Sadr R, Klewicki J (2003) A self-consistent model for the saturation dynamics of the vortex shedding around the mean flow in the unstable cylinder wake. Phys Fluids 15:1233-1246

Segalini A, Talamelli A (2011) Experimental analysis of dominant instabilities in coaxial jets. Phys Fluids 23:024103

Sieber M, Paschereit CO, Oberleithner K (2016) Spectral proper orthogonal decomposition. J Fluid Mech 792:798-828

Sirovich L (1987) Turbulence and the dynamics of coherent structures. part i: Coherent structures. Q Appl Math 45:561-571

Tiwari S, Bale S, Patwardhan A, Nandakumar K, Joshi J (2019) Insights into the physics of dominating frequency modes for flow past a stationary sphere: Direct numerical simulations. Phys Fluids 31:045108

Tomboulides A, Orszag S (2000) Numerical investigation of transitional and weak turbulent flow past a sphere. J Fluid Mech 416:45-73

Vanierschot M (2019) The break of symmetry in annular sudden expansion flows. In: Presented at the 8th International Symposium on Bifurcations and Instabilities in Fluid Dynamics (BIFD), Limerick

Vanierschot M, Van den Bulck E (2011) Experimental study of low precessing frequencies in the wake of a turbulent annular jet. Exp Fluids 50:189-200

Vanierschot M, Persoons T, Van den Bulck E (2009) A new method for annular jet control based on cross-flow injection. Phys Fluids 21:025103

Vanierschot M, Van Dyck K, Sas P, Van den Bulck E (2014) Symmetry breaking and vortex precession in low-swirling annular jets. Phys Fluids 26:105110

Wawrzak K, Boguslawski A, Tyliszczak A, Saczek M (2019) Les study of global instability in annular jets. Int J Heat Fluid Flow 79:108460

Wieneke B (2008) Volume self-calibration for 3d particle image velocimetry. Exp Fluids 45:549-556

Publisher's Note Springer Nature remains neutral with regard to jurisdictional claims in published maps and institutional affiliations. 


\section{Authors and Affiliations}

\section{Maarten Vanierschot ${ }^{1}\left(\mathbb{D} \cdot\right.$ Mustafa Percin ${ }^{2} \cdot$ Bas W. van Oudheusden ${ }^{3}$}

1 Department of Mechanical Engineering, KU Leuven, Leuven, Belgium

2 Department of Aerospace Engineering, Middle East Technical University, Ankara, Turkey
3 Department of Aerospace Engineering, Delft University of Technology, Delft, the Netherlands 